\title{
Evolution in invasive plants: implications for biological control
}

\author{
Heinz Müller-Schärer ${ }^{1}$, Urs Schaffner ${ }^{1,2}$ and Thomas Steinger ${ }^{1}$ \\ ${ }^{1}$ Département de Biologie, Unité Ecologie et Evolution, Université de Fribourg, $\mathrm{CH}-1700$ Fribourg, Switzerland \\ ${ }^{2} \mathrm{CABI}$ Bioscience Centre, $\mathrm{CH}-2800$ Delémont, Switzerland
}

\begin{abstract}
Evidence is increasing that invasive plants can undergo rapid adaptive evolution during the process of range expansion. Here, we argue that evolutionary change during invasions will also affect plant-antagonist interactions and, thus, will have important implications for biological control programmes targeted at invasive plants. We explore how altered selection in the new range might influence the evolution of plant defence (resistance and tolerance) and life history. The degree to which such evolutionary processes might affect biological control efficacy is largely unexplored. We hope that, by testing the hypotheses that we propose here, a closer link can be established between biological control and evolutionary biology, to the benefit of both disciplines.
\end{abstract}

BIOLOGICAL INVASIONS (see Glossary) by flowering plants have become more important as a result of increasing anthropogenic activities that affect species dispersal (through trade and travel) and habitat susceptibility (through changes in disturbance regimes) [1]. Although only a few EXотIC plant species invade natural communities and develop high population densities, those that do so pose one of the most serious threats to biodiversity [2]. Two contrasting, but mutually non-exclusive hypotheses address the relationship between phenotype and invasion success; the first assumes that invaders are pre-adapted with traits that make them successful invaders, whereas the second postulates successful invasion as the outcome of rapid evolutionary change once the species has become established in the new habitat [3].

Adaptation to local conditions is an important form of evolution in plant populations because plants are sessile. Many of the best examples of rapid evolution involve invasive plants [4], possibly because of the genetic processes involved during invasion that enhance genetic variation (on which selection can act; including hybridization) (e.g. [5]), or to strong directional selection exerted by abiotic and/or biotic factors. Interactions with competitors and natural enemies differ strongly between the native and introduced range [6-8]. If evolutionary change does occur, knowledge of its pace and direction might help to improve our predictions of the impact of subsequent BIOLOGICAL CONTROL attempts. The CLASSICAL approach

Corresponding author: Heinz Müller-Schärer (heinz.mueller@unifr.ch). Available online 4 June 2004 of biological control has been the most successful control strategy against invaders and is currently the curative control measure of choice against environmental weeds, owing to its effectiveness, low cost and relatively high environmental safety ([2,9], but see [10]).

The rate of adaptive evolution is determined by two components: selection and heritable variation. Therefore, to understand adaptive evolution in invasive plants, we must investigate how selection pressures on phenotypes differ between the native and the introduced range, and how the invasion process affects the amount of genetic variation expressed in a population. Here, we explore potential evolutionary trajectories of plant traits that are associated with herbivore defence and plant life history in the new range, and present hypotheses about how these changes might influence the efficacy of biological control. Recent developments in evolutionary theory, quantitative genetics and molecular tools offer a new integral approach with which to investigate such phenotypic evolution.

\section{Glossary}

Allelopathic effects: designate negative effects of one plant on another one through the release of chemical compounds into the environment.

Biological control: the use of natural enemies to control invasive species; classical biological control refers specifically to the introduction of a specialist exotic enemy to control an invasive exotic species.

Biological invasions: disruption of natural communities and ecosystems by an increase in distribution and abundance of exotic species.

Exotic: a species that has been dispersed outside of its native range as a result of human-mediated transportation.

Monocarpic (or semelparous plants): individuals arising from a seed that die after flowering; polycarpic or iteroparous plants can flower more than once. Qualitative defences: defences that include secondary plant metabolites, such as alkaloids and glucosinolates, which usually occur at low concentrations and are toxic to many generalist herbivores. Adapted specialized herbivores might use these chemicals to find their host plant and to sequester them for their own defence.

Quantitative defences: defences that are based on digestibility-reducing chemicals that are derived from the primary (e.g. cellulose) or secondary plant metabolism (e.g. tannins), and that usually occur in high concentration and act in a dosage-dependent fashion; defend a plant against specialist herbivores and those generalist herbivores that have adapted to the plant toxins. Resistance: mechanism whereby a plant minimizes the amount of damage it experiences; includes production of secondary chemicals, trichomes, and phenological patterns that enable escape from damage. Resistance traits are expected to reduce the preference or performance of herbivores, and thus to produce reciprocal selection on herbivores and lead to coevolution.

Specialist enemy: attacks only a single species (monophagous) or a few closely related species (oligophagous), as opposed to generalist enemies, which attack a wide range of species (polyphagous).

Tolerance: buffers negative effects of natural enemies on fitness through compensatory growth and reproduction after damage; generally thought to have no effect on herbivores. 


\section{Plant traits under novel selection in the new range}

In its native range, a plant species is attacked by an array of natural enemies, including mammals, insects and molluscs, and fungal, bacterial and viral pathogens. Some of these have a narrow host range (SPECIALIST ENEMIES), whereas others can attack many plant species (GENERALIST ENEMIES). The enemy release hypothesis posits that introduced plants experience a decrease in regulation by herbivores and other natural enemies and, therefore, can spread rapidly $[7,11]$. It has been shown that invasive exotic plants do suffer a reduced overall amount of herbivore damage [6], or are attacked by fewer herbivorous invertebrates [7], fungal and viral pathogens [8] compared with the same species in their native range.

The impoverished herbivore communities of plants in a new region can be primarily attributed to the lack of specialist enemies [7]. However, the level of attack by local generalists can vary considerably $[11,12]$ and can even hinder the invasion of certain communities (the biotic resistance hypothesis; see [11]), such as tropical forests, where lethal attack by native insects has repeatedly prevented the introduction of economically important tree genera [13]. Therefore, we propose that the most prominent change experienced by introduced plants in terms of natural enemies is a shift in the composition toward an assemblage that is dominated by generalists. Attack by specialists is restricted to those cases in which natural enemies feeding on closely related natives colonize the introduced plant, or where specialist herbivores have been co-introduced [7]. The shift in the composition of the natural enemy complex in the exotic range, along with changes in the competitive environment and in resource availability [14], is expected to incur altered selection on traits in invasive plants. Here, we explore how altered selection in the new range might influence the evolution of plant defence (RESISTANCE and TOLERANCE) and life cycle, and how evolutionary change might affect biological control efficacy.

\section{Resistance}

Based on optimal defence theory [15], the evolution of increased competitive ability (EICA) hypothesis proposed by Blossey and Nötzold [16] predicts that invasive plants will shift their resource allocation from herbivore defence to increased growth or reproduction because of the absence of herbivores in the new range. Here, we argue that, because invasive plants are not completely released from herbivores, the EICA hypothesis needs refining to take into account the proposed shift in the herbivore complex toward an assemblage that is dominated by generalists.

The EICA hypothesis assumes that plant defence chemicals incur fitness costs. For QUALITATIVE DEFENCE [15] (toxins such as alkaloids and glucosinolates), only a few of the many experimental studies on this topic found significant direct (allocation) costs (reviewed in [17]). By contrast, QUANTITATIVE DEFENCE compounds (e.g. lignins and tannins) appear to incur high costs because they constrain the inherent relative growth rate of plants [18] (Figure 1). For example, high levels of lignins reduce leaf palatability [19], but also specific leaf area (leaf area per

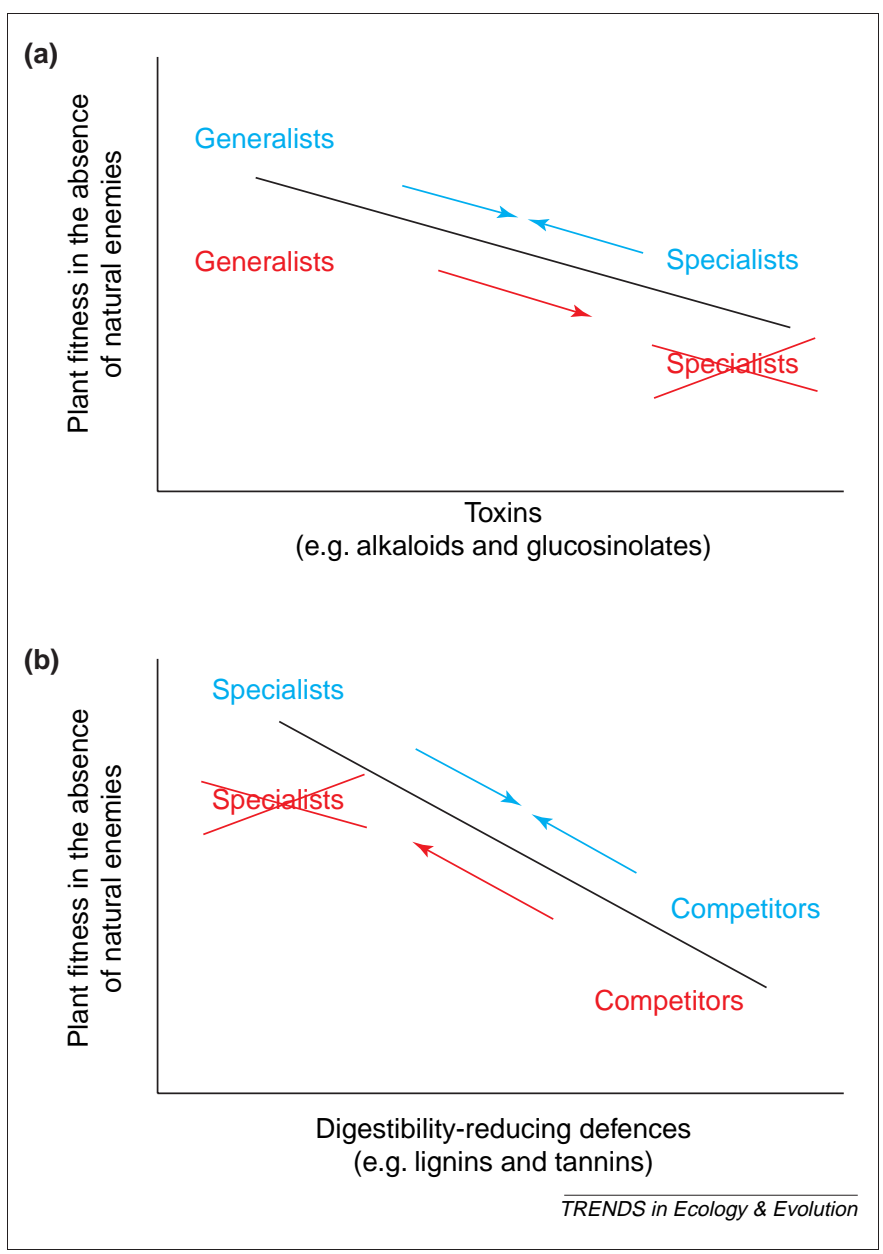

Figure 1. Differential selection on plant resistance traits in the native and introduced range. Fitness functions of (a) toxins (qualitative defences) and (b) digestibility-reducing defences (quantitative defences) in the absence of antagonists (black lines). The assumed higher costs of quantitative, relative to qualitative defences is indicated by the steeper fitness function. In the native range (in blue), qualitative defences are expected to be maintained at intermediate levels owing to opposing selection exerted by specialist and generalist herbivores (a). Quantitative defences, however, are expected to be maintained at intermediate levels owing to opposing selection exerted primarily by specialist herbivores and plant competitors (b). Assuming that invasive plants experience at least moderate attack by native generalist herbivores, but are largely free of attack by specialist herbivores, concentrations of plant toxins should therefore increase in the introduced range (in red), rather than decrease as predicted by the evolution of increased competitive ability (EICA) hypothesis (a). By contrast, relaxation from attack by specialist herbivores in the introduced range is expected to select for lower levels of quantitative defences, which might be reallocated to increase competitive ability (b), as proposed by the EICA hypothesis.

leaf mass), one of the main determinants of relative growth rate $[18,20]$.

In the native range, different antagonists are expected, based on optimal defence theory, to impose differential selection on both the qualitative and quantitative defence traits of plants (Figure 1). First, high concentrations of plant toxins might deter generalists while attracting specialists that either use them as a cue to locate or to accept the host plant for oviposition and/or feeding or that sequester the toxins and use them for their own defence $[21,22]$. For example, iridoid glycosides are toxic to many generalist herbivores, but Nieminen et al. found that individual plants of Plantago lanceolata with high iridoid glycoside concentrations in the field were used for oviposition by the specialist fritillary butterfly Melithaea cinxia significantly more than were those plants with low 
concentrations [23]. The iridoid-sequestering larvae feeding on plants with high levels of iridoid glycosides also had lower rates of parasitism [23]. As a result of opposite selection imposed by specialist and generalist herbivores, we expect plants to evolve intermediate levels of toxins (Figure 1a), an expectation that is supported by a theoretical model [21].

Second, plant species or genotypes that have lower quantitative defence are more susceptible to specialists and to those generalists that have adapted to the toxins of the plant [24], but, as mentioned above, such defences constrain the growth rate of the plant [18]. Furthermore, interspecific studies revealed relative growth rate to be positively correlated with competitive ability and risk of invasiveness $[20,25]$. Thus, in the native range, selection exerted primarily by specialist natural enemies and plant competitors is expected to favour intermediate levels of quantitative defences (Figure 1b).

In the introduced range, where specialist herbivores are largely absent, but plants might be attacked by native generalist herbivores, we expect that plant toxins will increase in concentration ( $c f$. J. Joshi and K. Vrieling, pers. commun.), rather than decrease as predicted by the EICA hypothesis (Figure 1a). This is because high concentrations of toxins no longer have fitness costs associated with attracting or increasing the survival of specialist herbivores. Furthermore, there is increasing evidence that some plant toxins, including glucosinolates, also function to benefit plants in competitive interactions through ALLELOPATHIC EFFECTS [22,26]. Thus, an increase in their concentration might further enhance the potential of a population to become invasive. However, concentrations of lignins and/or tannins are expected to decrease, particularly when generalists in the introduced range are repelled by the plant toxins.

Therefore, we propose that the evolved increased vigour documented in the introduced range for a few plant species [3] is best explained by a reallocation of resources from costly quantitative defences to growth (Figure 1b), whereas toxin concentrations might well evolve in the opposite direction than that predicted by the EICA hypothesis (Figure 1a). We suggest that such plants will be particularly amenable to biological control, because target weeds with increased digestibility are likely to support a faster population build-up of specialist biocontrol agents.

The results from studies comparing herbivore resistance levels of native versus invasive plant populations, or the preference and/or performance of specialist versus generalist herbivores, are largely in agreement with the predictions of our hypothesis. So far, reduced levels of plant defence chemicals in the introduced range have only been reported for quantitative defence traits (phenolics [27] and tannins [28]). Accordingly, a few specialist herbivores showed increased survival or growth on plants from the introduced range, whereas generalists feeding on the same plants did not ([3], and references therein, [29]). Contrary to our predictions, Lankau et al. [30] report more feeding by generalist herbivores on introduced than on native genotypes of the Chinese tallow tree, Sapium sebiferum in some experiments, but similar chewing damage in other experimental settings.
We acknowledge that, to make our arguments transparent, we strongly rely on the generalizations and assumptions of the optimal defence theory, which have been disputed recently (reviewed in [15]). Nevertheless, we adhere to the basic idea that different defences act against different herbivores, and that invaders might lose some, but not all, of their defences. The evolutionary dynamics are also likely to vary among populations, depending, for example, on the level of attack by specialist versus generalist natural enemies in the recipient community, and the degree to which antiherbivore toxins might also function allelopathically. Rigorous examinations of our proposed refinement of the EICA hypothesis should compare levels of herbivory by specialist versus generalist herbivores in invasive versus native populations, and examine herbivore-mediated selection on plant toxins and digestibility-reducing compounds.

\section{Tolerance}

In addition to resistance, plant populations can also evolve tolerance as a response to attack. This involves mechanisms that enable plants to regenerate and reproduce following grazing [31,32]. Evolutionary ecologists have only recently begun to study tolerance and its consequences for the coevolutionary dynamics of plantherbivore interactions [33]. In the context of plant invasion, tolerance has received little attention. What evolutionary trajectory do we expect in the introduced range where the intensity of competition and herbivory can be altered compared with the native range? Tolerance might be a defence mechanism that is particularly important for plant species that lack efficient chemical defence. Assuming that tolerance incurs fitness costs (reviewed in [17,32]), we might expect the evolution of decreased tolerance but increased competitive ability when plants are introduced into a new range, similar to that proposed by the EICA hypothesis for resistance. However, various factors might limit such an evolutionary response. First, selection imposed by generalist insect herbivores might be strong enough to maintain high tolerance in the introduced range. Second, it is likely that other stress and disturbance factors (e.g. fire, frost or drought), which also operate in the introduced range, will lead to the maintenance of tolerance to herbivory, because similar compensatory mechanisms might be activated after the destruction of apical meristems (i.e. the tip of a growing stem or branch) by various abiotic stress and disturbance factors [31]. Third, many introduced plants invade grassland habitats, where they are browsed by cattle [34]. Thus, although in such habitats invasive plants might face lower levels of insect herbivory compared with their native range, browsing by cattle might nevertheless be common and impose strong selection on plant tolerance.

Therefore, effective tolerance mechanisms could be common in plant invaders. We propose that this can help explain why most introductions of insect biological control agents exhibit only weak negative effects on their hosts $[2,9]$. It might further explain the resulting superabundance of some of the biological control agents over extended time periods [10] because tolerance, unlike resistance, is generally not expected to regulate the 
population dynamics of its consumers. This scenario could hold true for the example of the highly compensating nvasive forb Centaurea maculosa and its biological control agents introduced into North America. Two introduced gallflies remained host specific, but have failed to control populations of their host plant. As a result, these insects now occur in North America at a density of 3000 larvae $\mathrm{m}^{-2}$ [10], compared with a density of $\sim 30$ larvae $\mathrm{m}^{-2}$ in their native range in Europe.

We are aware of only two studies that compared levels of tolerance in native versus invasive plant populations $[29,35]$. In both species studied (Alliaria petiolata and Solidago canadensis), levels of tolerance were similar between native and introduced populations. In three interspecific studies comparing regrowth capacity and resource allocation pattern between an introduced and a native species (Agropyron desertorum-A. spicatum, Lonicera japonica-L. sempervirens and Sapium sebiferum-Celtis laevigata [36-38]), the invading species were less affected by herbivory because of increased levels of compensatory growth. Thus, the few available data support the hypothesis that introduction will not alter the level of tolerance, but clearly, more experimental work is needed to understand how altered patterns of selection might influence the evolution of plant tolerance during the invasion process.

\section{Life cycle}

Observations of several plant species (e.g. Cynoglossum officinale, Senecio jacobaea, Oenothera spp., Digitalis purpurea, Verbascum thapsus, Centaurea stoebe and Tripleurospermum perforatum) indicate a change from a prevalent MONOCARPIC habit in the native range of temperate Eurasia to a POLYCARPIC habit associated with the invasion process into North America ([3], and references therein). We propose that these observations can be explained by the release from specialized natural enemies that can kill plants that overwinter after seed set, such as endophagous (mining and galling) root-feeding herbivores.

Using a dynamic state variable model, Klinkhamer et al. addressed whether herbivores can select for a monocarpic life cycle [39]. Assuming that herbivores are attracted to bolting and flowering plants, the authors examined a range of models in which herbivores affected fecundity, survivorship and the proportional allocation of resources to storage. A monocarpic strategy was favoured when herbivory affected survival, because reduced survival decreased gains from future reproduction, so resources not spent on current reproduction are effectively wasted. Using realistic parameter values for $C$. officinale from the dunes in the western Netherlands, the model predicted an optimal life history that was close to monocarpic, as observed in the field (where C. officinale is exclusively monocarpic), with $99 \%$ of resources spent on current reproduction. In this system, the most important herbivore is the root-feeding biological control agent Mogulones cruciger, the summer generation of which attacks almost exclusively flowering plants and destroys their roots. In support of our argument, $\sim 10 \%$ of all $C$. officinale were polycarpic in an experimental garden in Holkham (UK), where the weevil is absent (P. Klinkhamer, pers. commun.).

Thus, there is at least circumstantial evidence that reproduction-related mortality caused by herbivores can lead to a monocarpic habit. Further work needs to demonstrate the genetic basis of this trait variation as opposed to phenotypic plasticity. Besides herbivory, many other factors differing between the introduced and native range could explain the observed changes in life history (e.g. resources, climate or disturbance levels). Successful biological control programs offer the opportunity to test our herbivory hypothesis ( $c f$. Box 1 and 2).

\section{Conclusions}

We propose that a shift in the composition of the natural enemy complex toward an assemblage that is dominated by generalists alters selection on invading plants as compared with selection on the species in their native habitat. Up to now, only a few studies have been designed

\section{Box 1. Herbivory and plant life history: two case studies}

\section{Centaurea maculosa}

Centaurea maculosa, introduced into North America during the late 19th century, is a predominantly short-lived, polycarpic tetraploid that is native to eastern Europe. According to Ochsman [42], C. maculosa in North America is generally a misnomer for $C$. stoebe ssp. micranthos. By contrast, the most abundant and widely distributed C. maculosa in western Europe (=C. stoebe ssp. stoebe [42]) is a diploid biennnial (a monocarpic plant that lives for more than one year) $[43,44]$. These diploid populations are also the main source of subsequent introductions of specialized root feeders into North America to control C. maculosa (e.g. $[43,44])$. The potential for high seed production early in life (most polycarpic tetraploids generally begin to flower in their first year), together with the polycarpic life cycle, might have favoured the observed spread of the tetraploid plants in North America, as shown by a basic population model [43]. The predominantly polycarpic life cycle might have been able to spread only in environments that were free of (specialized) rootherbivores.

Since 1982, four root-feeding insect herbivores have been introduced into North America in an attempt to fight the C. maculosa invasion [45]. A positive correlation between the time since root herbivores were released (or relative reduction in weed density following biocontrol introductions as a measure of the selective force imposed by the rood-feeders) and the percentage of diploids in the population (assuming a close relationship between the ploidy level and the reproductive habit) would support the hypothesis that root feeders select for a monocarpic habit (Box 2). Further studies need to address the prevalence of genetic variation in monocarpic versus polycarpic habit, as well as consequences of chromosome doubling and the associated switch to a polycarpic life cycle for the invasion success $[2,3]$.

\section{Insular woodiness}

Numerous island-inhabiting species of predominantly herbaceous angiosperm genera are woody shrubs and trees [46]. This well documented insular woodiness might also be understood in the light of herbivore absence (for other explanations, see [46]). We might hypothesize that the evolution of the woody perennial life form is partially a consequence of a change from a monocarpic to a polycarpic habit owing to relaxed selection from herbivory on the islands. Indeed, members of the genus Echium of continental Europe are all herbaceous and monocarpic, whereas 17 of the 20 Echium species on islands are polycarpic. All Echium species on islands are woody perennials and are descended from continental herbaceous ancestors [46]. 


\section{Box 2. Plant invasions and biological control: testing evolutionary hypotheses}

Hypothesis 1: Biological control will be most efficient when invasive populations have low genetic variation

We expect that the release of highly adapted biocontrol agents will enable fast population build-up and spread when invasive plant populations are genetically depauperate as a result of bottlenecks and inbreeding. However, biocontrol efficacy will be reduced if control agents encounter new (combinations of) resistance traits in the new range $[47,48]$. This could be due to population mixing of previously isolated populations through multiple introductions of genetically highly structured source populations from the native range, or following interspecific hybridization between introduced and either native or introduced taxa [3]. In this case, release of agents from different host populations should be envisaged.

Hypothesis 2. Plants that have evolved increased vigour in the exotic range will experience a particularly fast population build-up of biocontrol agents

Assuming that increased vigour has evolved at the expense of quantitative defences, we expect these plants to support increased survivorship, larval developmental rates or fecundity of specialist biocontrol agents. In addition, increased levels of toxins in response to selection by generalist herbivores might benefit host finding and acceptance by specialist insects that use them as a cue to locate their host plant.

Hypothesis 3. The impact of biocontrol herbivores on plant performance will depend on the levels of resistance and tolerance evolved during the invasion process in the absence of specialist herbivores

In plant species where tradeoffs exist between tolerance and resistance [49,50], we predict both higher per-capita and overall impact of biological control agents on plant genotypes that are chemically defended by toxins, and lower impact on the tolerant genotypes.

Hypothesis 4. Selection in the introduced range, where specialist root-feeders that are attracted to flowering stems are absent will favour genotypes with a polycarpic life cycle over monocarpic genotypes

The release of biocontrol herbivores that selectively kill larger plants that overwinter after seed set (Box 1 ) is likely to reverse an evolutionary shift towards a polycarpic life cycle, and favour monocarpic genotypes. Thus, if evolution of polycarpism was the key to the invasiveness of a species, then that species should be especially susceptible to biocontrol.

to test specifically for the post-introduction evolution of traits related to plant defence. Clearly, identifying natural selection as the driving force leading to trait divergence between native and introduced populations is not a simple task, because other processes, such as founder effects and genetic drift, are equally likely to cause differences in phenotypic traits [40]. Recent developments in molecular marker techniques and statistical analyses offer great opportunities to disentangle these processes (e.g. by enabling the identification of source populations in the native range that can then be used for comparisons with introduced populations).

We are well aware that predicting consequences of evolutionary change in invasive plants for plantherbivore interactions would involve unravelling the innumerable processes, from changes in gene frequencies to plant fitness, population dynamics and community interactions. Therefore, testing the proposed hypotheses (Box 2) will not directly lead to making the outcome of biological control more predictive, but we hope to have indicated interesting studies that address important processes at specific levels of integration. Much has been learned in the past few decades about the effect of specialist herbivores on plant population dynamics from biological control programmes [41]. It is time to acknowledge that plant invasions and classical biological control programmes are also models with which to study evolution. We believe that a closer link between biological control and evolutionary biology can offer new insights and advance both disciplines.

\section{Acknowledgements}

We thank Ray Callaway, Dieter Ebert, Jasmin Joshi, John Maron, Peter McEvoy, Nigel Paul, Dean Pearson, Daniel Prati, Bernhard Schmid, Andy Sheppard, Klaas Vrieling and three anonymous referees for stimulating discussions or comments. The study was partly supported by the Swiss National Science Foundation through grant numbers 31-65356.01 to H.M-S. and 31-67044.01 to T.S., and through the National Centre of Competence in Research (SNSF-NCCR) Plant Survival.

\section{References}

1 Pimentel, D. et al. (2000) Environmental and economic costs of nonindigenous species in the United States. Bioscience 50, 53-65

2 Myers, J.H. and Bazely, D.R. (2003) Ecology and Control of Introduced Plants, Cambridge University

3 Müller-Schärer, H. and Steinger, T. (2004) Predicting evolutionary change in invasive, exotic plants and its consequences for plantherbivore interactions. In Genetics, Evolution and Biological Control (Ehler, L.E. et al., eds), pp. 137-162, CAB International

4 Reznick, D.N. and Ghalambor, C.K. (2001) The population ecology of contemporary adaptations: what empirical studies reveal about the conditions that promote adaptive evolution. Genetica 112-113, $183-198$

5 Ellstrand, N.C. and Schierenbeck, K.A. (2000) Hybridization as a stimulus for the evolution of invasiveness in plants? Proc. Natl. Acad. Sci. U. S. A. 97, 7043-7050

6 Wolfe, L.M. (2002) Why alien invaders succeed: support for the escape-from-enemy hypothesis. Am. Nat. 160, 705-711

7 Keane, R.M. and Crawley, M.J. (2002) Exotic plant invasions and the enemy release hypothesis. Trends Ecol. Evol. 17, 164-170

8 Mitchell, C.E. and Power, A.G. (2003) Release of invasive plants from fungal and viral pathogens. Nature 421, 625-627

9 Crutwell-McFadyen, R.E.C. (1998) Biological control of weeds. Annu. Rev. Entomol. 43, 369-393

10 Pearson, D.E. and Callaway, R.M. (2003) Indirect effects of hostspecific biological control agents. Trends Ecol. Evol. 18, 456-461

11 Maron, J.L. and Vilà, M. (2001) When do herbivores affect plant invasions? Evidence for the natural enemies and biotic resistance hypothesis. Oikos $95,361-373$

12 Agrawal, A.A. and Kotanen, P.M. (2003) Herbivores and the success of exotic plants: a phylogenetically controlled experiment. Ecol. Lett. 6, $712-715$

13 Mack, R.N. (1996) Predicting the identity and fate of plant invaders: emergent and emerging approaches. Biol. Conserv. 78, 107-121

14 Alpert, P. et al. (2000) Invasiveness, invasibility and the role of environmental stress in the spread of non-native plants. Perspect. Plant Ecol. Evol. Syst. 3, 52-66

15 Stamp, N. (2003) Out of the quagmire of plant defence hypotheses. Q. Rev. Biol. 78, 23-54

16 Blossey, B. and Nötzold, R. (1995) Evolution of increased competitive ability in invasive nonindigenous plants: a hypothesis. J. Ecol. 83, 887-889

17 Strauss, S.Y. et al. (2002) Direct and ecological costs of resistance to herbivory. Trends Ecol. Evol. 17, 278-285

18 Poorter, H. and de Jong, R. (1999) A comparison of specific leaf area, chemical composition and leaf construction costs of field plants from 15 habitats differing in productivity. New Phytol. 143, 163-176 
population dynamics of its consumers. This scenario could hold true for the example of the highly compensating nvasive forb Centaurea maculosa and its biological control agents introduced into North America. Two introduced gallflies remained host specific, but have failed to control populations of their host plant. As a result, these insects now occur in North America at a density of 3000 larvae $\mathrm{m}^{-2}$ [10], compared with a density of $\sim 30$ larvae $\mathrm{m}^{-2}$ in their native range in Europe.

We are aware of only two studies that compared levels of tolerance in native versus invasive plant populations $[29,35]$. In both species studied (Alliaria petiolata and Solidago canadensis), levels of tolerance were similar between native and introduced populations. In three interspecific studies comparing regrowth capacity and resource allocation pattern between an introduced and a native species (Agropyron desertorum-A. spicatum, Lonicera japonica-L. sempervirens and Sapium sebiferum-Celtis laevigata [36-38]), the invading species were less affected by herbivory because of increased levels of compensatory growth. Thus, the few available data support the hypothesis that introduction will not alter the level of tolerance, but clearly, more experimental work is needed to understand how altered patterns of selection might influence the evolution of plant tolerance during the invasion process.

\section{Life cycle}

Observations of several plant species (e.g. Cynoglossum officinale, Senecio jacobaea, Oenothera spp., Digitalis purpurea, Verbascum thapsus, Centaurea stoebe and Tripleurospermum perforatum) indicate a change from a prevalent MONOCARPIC habit in the native range of temperate Eurasia to a POLYCARPIC habit associated with the invasion process into North America ([3], and references therein). We propose that these observations can be explained by the release from specialized natural enemies that can kill plants that overwinter after seed set, such as endophagous (mining and galling) root-feeding herbivores.

Using a dynamic state variable model, Klinkhamer et al. addressed whether herbivores can select for a monocarpic life cycle [39]. Assuming that herbivores are attracted to bolting and flowering plants, the authors examined a range of models in which herbivores affected fecundity, survivorship and the proportional allocation of resources to storage. A monocarpic strategy was favoured when herbivory affected survival, because reduced survival decreased gains from future reproduction, so resources not spent on current reproduction are effectively wasted. Using realistic parameter values for $C$. officinale from the dunes in the western Netherlands, the model predicted an optimal life history that was close to monocarpic, as observed in the field (where C. officinale is exclusively monocarpic), with $99 \%$ of resources spent on current reproduction. In this system, the most important herbivore is the root-feeding biological control agent Mogulones cruciger, the summer generation of which attacks almost exclusively flowering plants and destroys their roots. In support of our argument, $\sim 10 \%$ of all $C$. officinale were polycarpic in an experimental garden in Holkham (UK), where the weevil is absent (P. Klinkhamer, pers. commun.).

Thus, there is at least circumstantial evidence that reproduction-related mortality caused by herbivores can lead to a monocarpic habit. Further work needs to demonstrate the genetic basis of this trait variation as opposed to phenotypic plasticity. Besides herbivory, many other factors differing between the introduced and native range could explain the observed changes in life history (e.g. resources, climate or disturbance levels). Successful biological control programs offer the opportunity to test our herbivory hypothesis ( $c f$. Box 1 and 2).

\section{Conclusions}

We propose that a shift in the composition of the natural enemy complex toward an assemblage that is dominated by generalists alters selection on invading plants as compared with selection on the species in their native habitat. Up to now, only a few studies have been designed

\section{Box 1. Herbivory and plant life history: two case studies}

\section{Centaurea maculosa}

Centaurea maculosa, introduced into North America during the late 19th century, is a predominantly short-lived, polycarpic tetraploid that is native to eastern Europe. According to Ochsman [42], C. maculosa in North America is generally a misnomer for $C$. stoebe ssp. micranthos. By contrast, the most abundant and widely distributed C. maculosa in western Europe (=C. stoebe ssp. stoebe [42]) is a diploid biennnial (a monocarpic plant that lives for more than one year) $[43,44]$. These diploid populations are also the main source of subsequent introductions of specialized root feeders into North America to control C. maculosa (e.g. $[43,44])$. The potential for high seed production early in life (most polycarpic tetraploids generally begin to flower in their first year), together with the polycarpic life cycle, might have favoured the observed spread of the tetraploid plants in North America, as shown by a basic population model [43]. The predominantly polycarpic life cycle might have been able to spread only in environments that were free of (specialized) rootherbivores.

Since 1982, four root-feeding insect herbivores have been introduced into North America in an attempt to fight the C. maculosa invasion [45]. A positive correlation between the time since root herbivores were released (or relative reduction in weed density following biocontrol introductions as a measure of the selective force imposed by the rood-feeders) and the percentage of diploids in the population (assuming a close relationship between the ploidy level and the reproductive habit) would support the hypothesis that root feeders select for a monocarpic habit (Box 2). Further studies need to address the prevalence of genetic variation in monocarpic versus polycarpic habit, as well as consequences of chromosome doubling and the associated switch to a polycarpic life cycle for the invasion success $[2,3]$.

\section{Insular woodiness}

Numerous island-inhabiting species of predominantly herbaceous angiosperm genera are woody shrubs and trees [46]. This well documented insular woodiness might also be understood in the light of herbivore absence (for other explanations, see [46]). We might hypothesize that the evolution of the woody perennial life form is partially a consequence of a change from a monocarpic to a polycarpic habit owing to relaxed selection from herbivory on the islands. Indeed, members of the genus Echium of continental Europe are all herbaceous and monocarpic, whereas 17 of the 20 Echium species on islands are polycarpic. All Echium species on islands are woody perennials and are descended from continental herbaceous ancestors [46]. 